\title{
The hadronic interaction model Sibyll 2.3c and Feynman scaling
}

\section{Felix Riehn}

LIP Lisbon, Av. Prof. Gama Pinto 2, 1649-003 Lisbon, Portugal

E-mail: friehn@lip.pt

\section{Hans P. Dembinski}

Max Planck Institute for Nuclear Physics, Postfach 103980, 69029 Heidelberg, Germany

\section{Ralph Engel*}

Karlsruher Institut für Technologie, Institut für Kernphysik, Postfach 3640, 76021 Karlsruhe,

Germany

\section{Anatoli Fedynitch}

DESY, Platanenallee 6, 15738 Zeuthen, Germany

\section{Thomas K. Gaisser}

Bartol Research Institute, Department of Physics and Astronomy, University of Delaware, Newark, DE 19716, USA

\section{Todor Stanev}

Bartol Research Institute, Department of Physics and Astronomy, University of Delaware, Newark, DE 19716, USA

\begin{abstract}
The Monte Carlo model Sibyll has been designed for efficient simulation of hadronic multiparticle production up to the highest energies as needed for interpreting cosmic ray measurements. For more than 15 years, version 2.1 of Sibyll has been one of the standard models for air shower simulation. Motivated by data of LHC and fixed-target experiments and a better understanding of the phenomenology of hadronic interactions, we have developed an improved version of this model, version 2.3, which has been released in 2016. In this contribution we present a revised version of this model, called Sibyll $2.3 \mathrm{c}$, that is further improved by adjusting particle production spectra to match the expectation of Feynman scaling in the fragmentation region. After a brief introduction to the changes implemented in Sibyll 2.3 and $2.3 \mathrm{c}$ with respect to Sibyll 2.1, the current predictions of the model for the depth of shower maximum, the number of muons at ground, and the energy spectrum of muons in extensive air showers are presented.
\end{abstract}

35th International Cosmic Ray Conference

10-20 July, 2017

Bexco, Busan, Korea

\footnotetext{
*Speaker.
} 


\section{Introduction}

Sibyll is one of the standard hadronic event generators used in the simulation of extensive air showers initiated by high energy cosmic rays. It is designed to describe the general features of hadronic multiparticle production, like the leading particle effect, the formation of high- $p_{\mathrm{T}}$ jets predicted in QCD, the production of diffractively excited states of the projectile and target, and approximate scaling of leading particle distributions with interaction energy. In Sibyll, focus is put on those physics aspects that are most relevant for the development of extensive air showers, like energy flow and particle production in the forward phase space region. While the model is kept as simple as possible, the important microscopic physics concepts and the general principles of scattering theory and unitarity are implemented to allow extrapolation to energies and phase space regions beyond the reach of colliders [1-3].

In the recently released Sibyll 2.3 [4] the previous model version [3] was extended and updated while retaining the overall underlying picture of hadronic interactions implemented already in Sibyll 2.1. The most notable changes of Sibyll 2.3 wrt. version 2.1 are

- New fits to total and elastic cross sections for $p-p, \pi-p$ and $K-p$ interactions to match new LHC and fixed-target data.

- Explicit treatment of remnant excitations for better description of leading particle production.

- Enhanced production of vector resonances in the fragmentation region of mesons $\left(\rho^{0}\right)$.

- Implementation of diffraction dissociation in interactions of hadrons with nuclei based on a two-component model (ground state and excited state of projectile and target hadrons), similar to the Good-Walker model of diffraction [5].

- Increase of the rate of baryon-antibaryon pair production in string fragmentation, including a higher production rate in minijet fragmentation than in soft processes.

- Implementation of a phenomenological model for describing the production of charm particles.

A summary of the physics ideas and principles on which Sibyll is based can be found in Ref. [6] and a first description of Sibyll 2.3 is given in Ref. [4]. Here we will discuss the update of Sibyll 2.3 to $2.3 \mathrm{c}$.

\section{Scaling behavior of leading particle distributions and extrapolation to high energy}

One of the important tasks of interaction models used in astroparticle physics is the extrapolation from the energy range and phase space covered by laboratory measurements to energies and phase space regions that play a role in air shower development [7] or in the production of atmospheric leptons [8]. While measurements at LHC, for example, extend to $\sim 10 \mathrm{TeV}$ in $\mathrm{cm}$. energy, the interaction energies of cosmic rays, as they are studied by the Pierre Auger Observatory, extend up to $\mathrm{cm}$. energies of $400 \mathrm{TeV}$. In terms of phase space the situation is worse still. Interactions with small momentum transfers (low- $Q^{2}$, 'soft' scattering) are most common. These interactions cannot be calculated within perturbative $\mathrm{QCD}$, the currently best approach for quantitatively predicting hadronic particle production. Moreover, in such interactions, particles are emitted at very small angles, which means they escape detection through the beam pipe in particle collider experiments. 


\subsection{Feynman scaling and leading particles}

To allow for meaningful predictions, additional principles have to be invoked. One such principle is that of Feynman scaling [9]. It states that the production cross section of particle $i$ at high energy is independent of the interaction energy and only depends on transverse momentum and the longitudinal momentum fraction $x_{\mathrm{F}}=p_{z} / p_{z}^{\max }$

$$
E \frac{\mathrm{d}^{3} \sigma_{i}}{\mathrm{~d}^{3} \vec{p}} \rightarrow f_{i}\left(p_{\mathrm{T}}, x_{\mathrm{F}}\right)
$$

While Feynman scaling was quickly found to be violated by jet formation in hard scattering processes [10], it is still believed to hold approximately in the so-called fragmentation region where soft interactions dominate. Translated into longitudinal phase space, one expects a universal shape for the production cross section of a given particle for $x_{\mathrm{F}} \gtrsim 0.1$ at high energy. Assuming Feynman scaling, the production spectra in an interaction model can be tuned to measurements at low energy where full phase space coverage is still feasible and then reliably extrapolated to high energies.

In parton-based interaction models such as Sibyll, Feynman scaling is not imposed on the hadron level. Instead, the momentum distributions of the partons at the string ends and, correspondingly, of the hadrons produced in string fragmentation satisfy Feynman scaling. Due to overall energy-momentum conservation and the rapid increase of the secondary particle multiplicity, violation of Feynman scaling of leading particles is ultimately expected at very high energy. It is an open question to what extent Feynman scaling might be violated for leading particles at intermediate energies. In the limit of black disc scattering even a total absence of a leading particle effect, and hence maximum violation of Feynman scaling of the leading particles, can be expected [11].

Sibyll $2.3 \mathrm{c}$ has been tuned to represent the most conservative assumption, namely minimal violation of Feynman scaling of leading particle distributions. Other models (EPOS [12], QGSjet [13, 14]) show a stronger violation of Feynman scaling.

\subsection{Sibyll predictions for leading particle distributions}

As an example for Feynman scaling, the production spectrum of $\pi^{-}$for different energies in Sibyll 2.1 is shown in Fig. 1 (left). As the $\mathrm{cm}$. energy increases, more and more particles are produced in the so-called central region $\left(x_{\mathrm{F}}<0.1\right.$, large- $\left.p_{\mathrm{T}}\right)$, while the rest of the spectrum remains unchanged. As previously mentioned, Feynman scaling is not explicitly implemented in the model but is an emergent phenomenon. It is a direct, albeit not necessarily evident prediction of the naive parton model and factorization [9]. Adding conservation of energy, the increase in the central region is expected to result in some softening of the forward spectra.

In models that predict explicit violation of Feynman scaling in the forward region, parton distributions of soft and hard processes are connected (i.e. breaking factorization) by, for example, deriving both from a common initial state [14]. Due to the connection of the forward region to the central region in such models, the extrapolation is still well constrained for the entire phase space, even without Feynman scaling. Different assumptions are, however, possible and lead to different extrapolations to high energy. With the current understanding and existing accelerator data of leading particle production it is not possible to distinguish between different scenarios.

In Fig. 1 (right), the production spectrum for Sibyll 2.3 is shown. Contrary to the general expectation, the spectrum is shifted towards larger momentum fractions the higher the interaction 

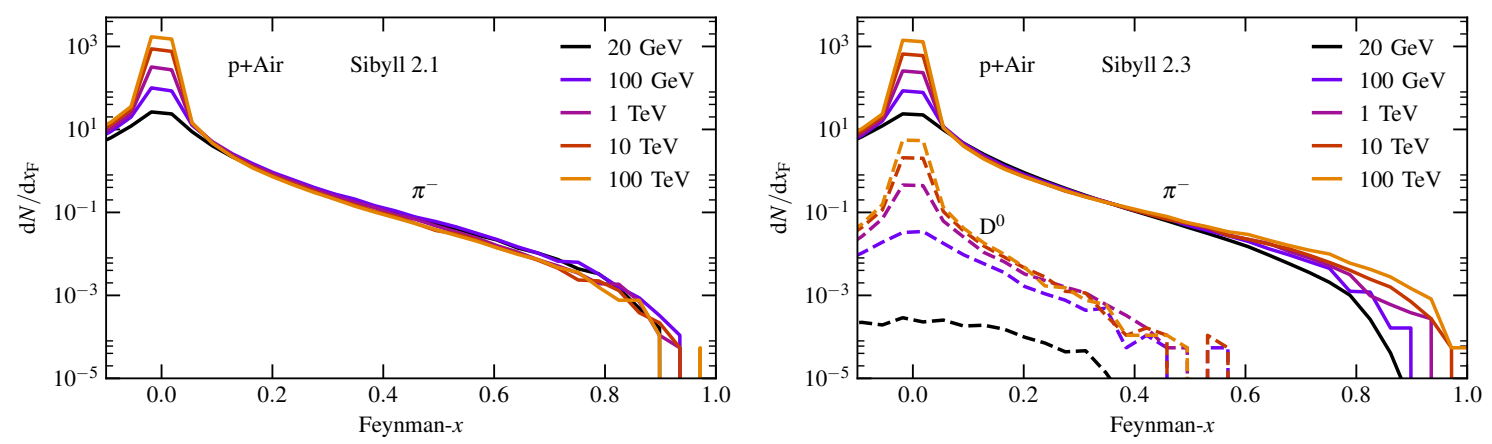

Figure 1: Spectrum of longitudinal momentum of $\pi^{-}$in Sibyll 2.1 (left) and Sibyll 2.3 (right). The hardening of the spectrum in Sibyll 2.3 is contrary to the expectation from Feynman scaling. For charmed hadrons (Sibyll 2.3 only) the scaling violations are not as prominent.

energies are. While the effect is not very pronounced, it shows that an undesired interplay between different processes responsible for leading particle production takes place.

At parton level, Feynman scaling emerges from the factorization of the different interaction scales. In addition the hadronization model used in Sibyll, which is the string fragmentation model $[15,16]$, has the property of preserving the scaling (assuming a constant string tension). One of the key differences between Sibyll 2.1 (scaling) and Sibyll 2.3 (no scaling) is the new treatment of excited beam remnants. While in Sibyll 2.1 the leading particles produced by the beam remnants are effectively included by adjusting the fragmentation process for partons related to the incoming hadrons (valence quarks), Sibyll 2.3 dynamically produces excited states whose decay products then emerge as the leading particles. Although this remnant model seems a good candidate to explain the unexpected scaling violations in the forward region in Sibyll 2.3, no direct connection was found.

Instead, the violations were found to be induced by an extension to string fragmentation that allows for the break-up of diquarks, the so-called popcorn model [17]. This model is used to reduce the correlation between baryon-antibaryon pairs in the fragmentation process, as observed in $e^{+} e^{-}$ annihilation. It also leads to a broader rapidity distribution and harder $x_{\mathrm{F}}$-spectrum. In combination with the fast diquark from an initial proton it results in a significant hardening of the meson spectra. In Fig. 2 (left) the restoration of the scaling behavior for Sibyll 2.3, if diquark break-up disabled, is demonstrated. The effect of reverting the explicit treatment of the remnant excitation to the effective treatment in Sibyll 2.1 is also shown. The large deviation from scaling at $20 \mathrm{GeV}$ is a lowenergy effect. It is related to the transition from string fragmentation to resonance production and decay. The popcorn mechanism was initially introduced to the model to improve this transition. In Sibyll 2.3c this is compensated by allowing larger remnant excitation masses and by retuning the fragmentation function and string tension. Furthermore, at low energy, the so-called associated production $p^{\star} \rightarrow \Lambda^{0}+\mathrm{K}^{+}$was enhanced by adding it as an explicit final state of the isotropic phase space decay (fireball).

The resulting spectrum is shown in Fig. 2 (right). Scaling behavior in the forward region is successfully restored. 

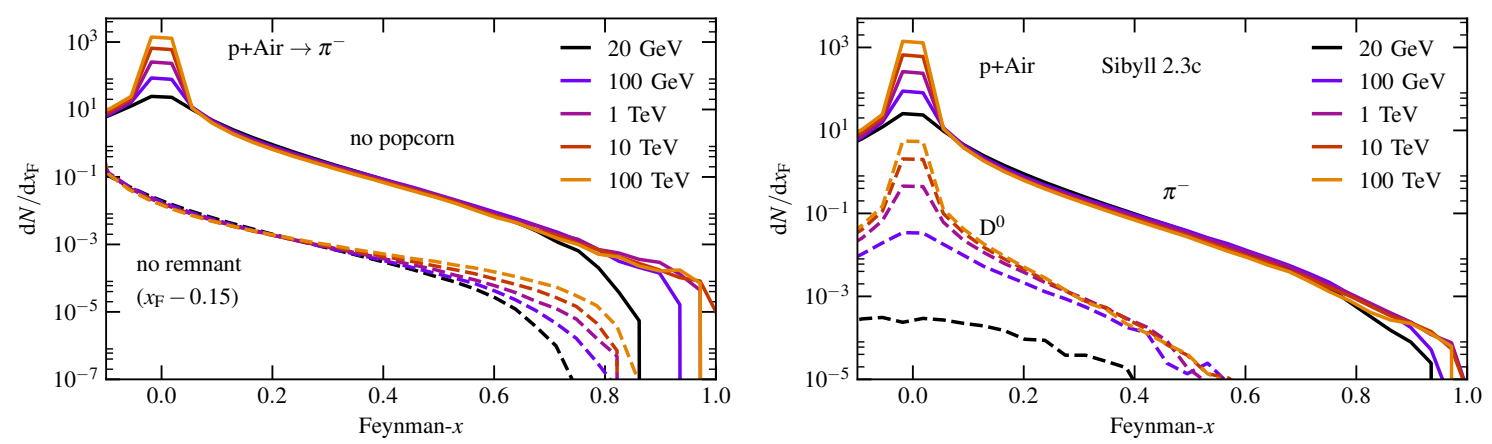

Figure 2: Spectrum of longitudinal momentum of pions and charmed hadrons in Sibyll 2.3 without 'popcorn' (left, solid lines), without the explicit remnant treatment (left, dashed lines) and in the retuned Sibyll 2.3c (right). The scaling violations in the fragmentation region in Sibyll 2.3 are induced by the interference of the popcorn mechanism with the leading quarks. The production of charmed hadrons in Sibyll $2.3 \mathrm{c}\left(\mathrm{D}^{0}\right.$, dashed lines on the right) is mostly unaffected because their production is dominated by central production.

\subsection{Kaon spectra and atmospheric lepton fluxes}

In addition to the interpretation of cosmic ray measurements, EAS simulations are equally important in the context of high energy neutrinos, since the bulk of cosmic rays produces a constant background flux of atmospheric neutrinos. Integration over the whole spectrum of cosmic rays shifts the weight of particle production into the forward fragmentation region [8]. The hardening of the longitudinal spectrum in Sibyll 2.3, therefore, has an even larger impact on the predictions of atmospheric fluxes of muons and neutrinos than its impact on EAS. In this context, kaons are of particular importance. Because of their shorter lifetime combined with the steep cosmic-ray spectrum, their contribution to the fluxes of atmospheric leptons increases above a $1 \mathrm{TeV}$. The effect is most important for $v_{\mu}$ where, because of the decay kinematics, kaons become the dominant parent. Figure 3 (left) illustrates the problem for Sibyll 2.3 by comparing its predictions separately for production of $\mathrm{K}^{+}$and $\mathrm{K}^{-}$to the kaon spectra measured by NA49 [18]. Production of $\mathrm{K}^{+}$is overestimated in Sibyll 2.3. (Note that a weight factor of $x_{\mathrm{F}}^{1.7}$ is applied to reflect the effect of the steep primary cosmic-ray spectrum.) The hard spectrum of $\mathrm{K}^{+}$is due to the same effect as the scaling violations discussed in the previous section, namely the promotion of mesons to leading particles by diquark break-up. This interpretation is confirmed by noting that the effect is not present for negatively charged kaons. This difference between the charge states is expected given the absence of strange valence quarks in the proton, so the negative charge state $\left(\mathrm{K}^{-}(\bar{u} s)\right)$ cannot be associated with leading quarks. With the adjustments ${ }^{1}$ in Sibyll 2.3c, the shape of the kaon spectrum is reproduced more accurately. At the same time the advantages of the explicit treatment of the remnant excitations are preserved, as is shown in the comparison with the energy spectrum of neutrons measured by LHCf [21] in Fig. 3 (right).

The predictions of Sibyll 2.3c for the atmospheric lepton fluxes are presented in a separate contribution at this conference [22].

\footnotetext{
${ }^{1}$ The hadronization parameters for the updated Sibyll $2.3 \mathrm{c}$ were found by fitting a parameterization of the event generator response to the pp measurements by NA49 using the Professor [19] and Rivet [20] tools.
} 

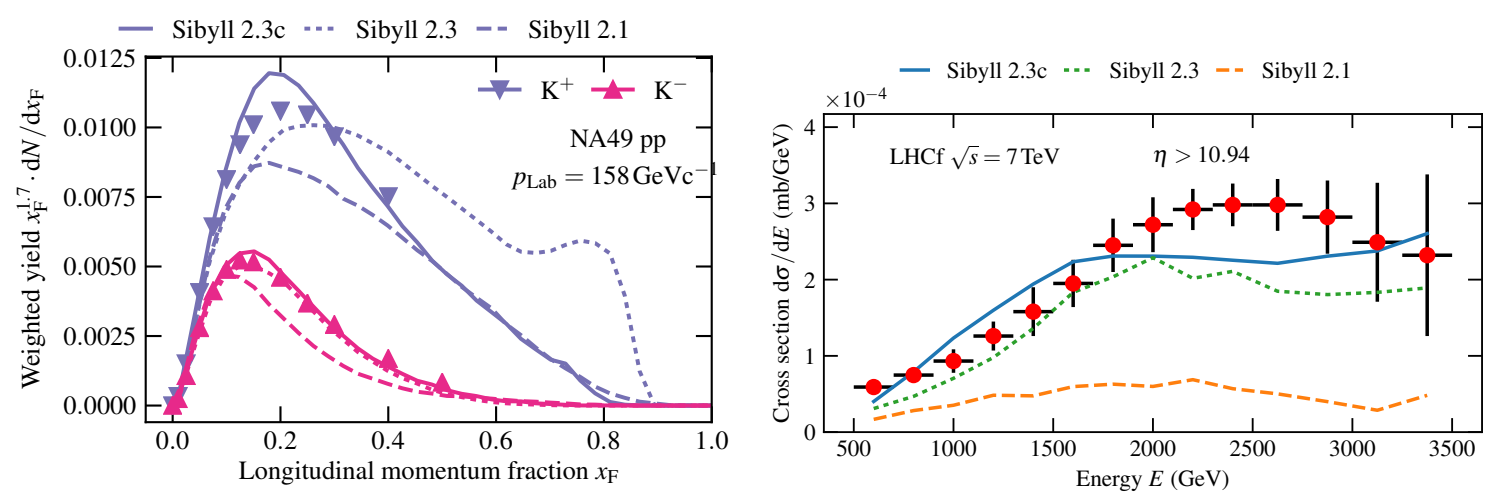

Figure 3: Left: Weighted spectra of kaon production in pp interactions [18]. Right: Energy spectrum of neutrons at small angles [21].

\section{Predictions for extensive air showers}

The update of the hadronic interaction model Sibyll presented here mainly concerns the evolution of the shape of the production spectra of mesons at large values of Feynman- $x$. Due to the weighting with the primary spectrum in the calculation of the atmospheric fluxes of leptons these changes at large $x_{\mathrm{F}}$ will have a larger effect on the atmospheric fluxes than on air shower predictions, where the production spectra enter via $x \mathrm{~d} \sigma / \mathrm{d} x$. In addition, kaons play a smaller role in air showers.

In the following we show predictions calculated with CONEX [23]. The energy threshold for the transition between the Monte Carlo and numerical cascade equation was set to $5 \%$ of the primary energy.

For air showers, the updated model Sibyll 2.3c predicts a slightly shallower depth of the average position of shower maximum $\left(X_{\max }\right)$ than Sibyll 2.3. Compared to Sibyll 2.1, $X_{\max }$ is deeper by $20 \mathrm{~g} / \mathrm{cm}^{2}$ across the energy range of ultra-high-energy cosmic rays, see Fig. 4 (left). The number of muons $\left(N_{\mu}\right)$ is larger than in Sibyll 2.3, albeit only by a very small amount (Fig. 4 (right)). Relative to Sibyll 2.1, $N_{\mu}$ is larger by a factor $\sim 1.35$ at $10^{16} \mathrm{eV}$ and by a factor $\sim 1.6$ at $10^{20} \mathrm{eV}$. In the current version, Sibyll predicts the largest number of muons of all post-LHC interaction models $[13,24]$. There are no new processes in Sibyll 2.3c wrt. Sibyll 2.3 that could cause the slight increase in muon number, the only change is the shape of the production spectra.

The effect on muon production is summarized by the change in the energy spectrum of muons (Fig. 5). The figure shows that the softening of the spectra and the restoration of Feynman scaling leads to the increase of muons with low-energy. Below $100 \mathrm{GeV}$ Sibyll $2.3 \mathrm{c}$ produces most muons of all models. Beyond $1 \mathrm{PeV}$ the contribution from the prompt decays of charmed hadrons sets in. The strong increase of the number of muons at low energies of the post-LHC models relative to Sibyll 2.1 is due to: (i) increased production of baryon-antibaryon pairs, (ii) the increased ratio $\pi^{ \pm}: \pi^{0}$ in pion interactions in forward direction, mainly stemming from the formation of leading $\rho^{0}$ resonances instead of leading $\pi^{0}$ mesons.

In Fig. 5 (right) the ratio of the energy spectrum of muons of iron and proton showers is shown. With the post-LHC models, the overall difference between proton and iron decreases. In contrast to the energy spectrum of muons for the individual nuclei, the models agree very well on 

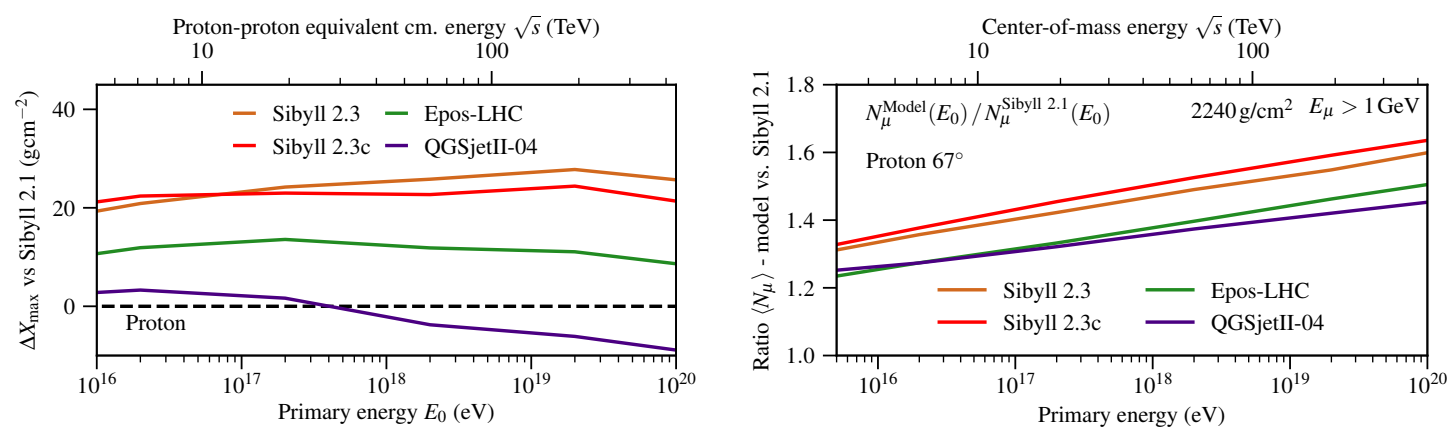

Figure 4: Difference between post-LHC models and Sibyll 2.1 for $X_{\max }$ (left) and $N_{\mu}$ (right).
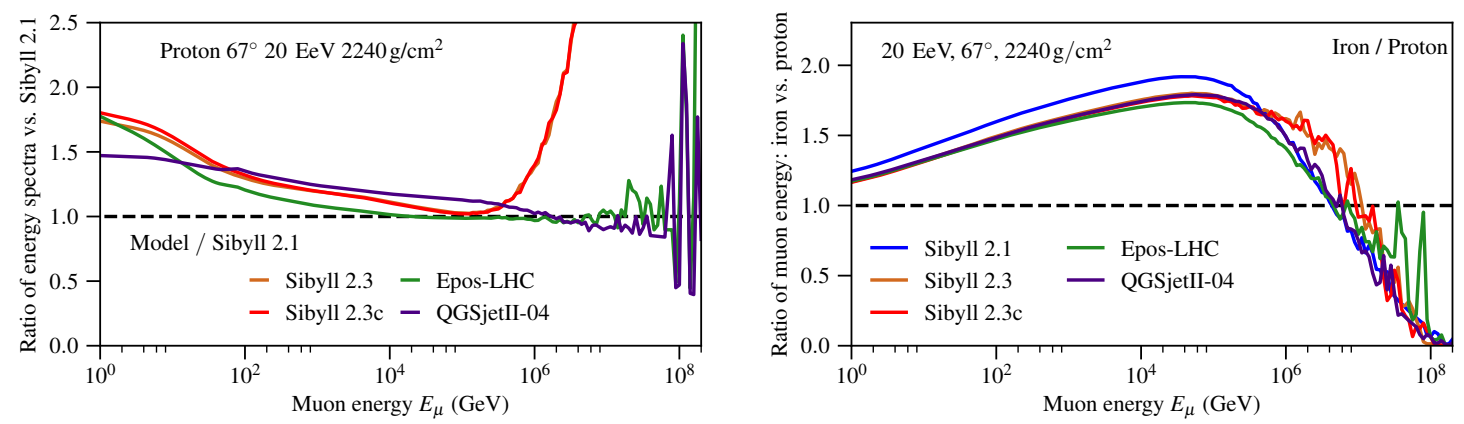

Figure 5: Ratio between the energy spectrum of muons for the post-LHC models and Sibyll 2.1 (left) and between different primaries (right). The primary energy is $10^{19} \mathrm{eV}$ and the spectra are evaluated at a depth of $2240 \mathrm{~g} / \mathrm{cm}^{2}$.

the difference between nuclei, in particular for muons of low energy.

\section{Conclusion \& Outlook}

We have developed a new version of Sibyll, called Sibyll 2.3c, by re-tuning the model version 2.3 to obtain a better description of NA49 data. It was found that Sibyll 2.3c approximately obeys Feynman scaling in the fragmentation region up to the highest energies and gives a better description of the measured kaon production spectra than previous versions. The predictions for extensive air showers are very similar to Sibyll 2.3, as are the predictions for production of charmed hadrons. Larger changes are found for inclusive fluxes of atmospheric leptons, which are discussed in [22].

Acknowledgements We thank our colleagues T. Pierog and S. Ostapchenko for many fruitful discussions and constructive criticism. This work is supported in part by the U.S. National Science Foundation (PHY-1505990) and in part by the KIT graduate school KSETA.

\section{References}

[1] J. Engel, T. K. Gaisser, T. Stanev, and P. Lipari Phys. Rev. D46 (1992) 5013-5025. 
[2] R. S. Fletcher, T. K. Gaisser, P. Lipari, and T. Stanev Phys. Rev. D50 (1994) 5710-5731.

[3] E.-J. Ahn, R. Engel, T. K. Gaisser, P. Lipari, and T. Stanev Phys. Rev. D 80 (2009) 094003, [0906.4113].

[4] F. Riehn, R. Engel, A. Fedynitch, T. K. Gaisser, and T. Stanev EPJ Web Conf. 99 (2015) 12001, [1502.06353].

[5] M. L. Good and W. D. Walker Phys. Rev. 120 (1960) 1857-1860.

[6] R. Engel, F. Riehn, A. Fedynitch, T. K. Gaisser, and T. Stanev EPJ Web Conf. 145 (2017) 08001.

[7] R. Engel, D. Heck, and T. Pierog Ann. Rev. Nucl. Part. Sci. 61 (2011) 467-489.

[8] T. K. Gaisser and M. Honda Ann. Rev. Nucl. Part. Sci. 52 (2002) 153-199, [hep-ph/ 0203272 ].

[9] R. P. Feynman Phys. Rev. Lett. 23 (1969) 1415-1417.

[10] UA5 Collaboration, G. J. Alner et al. Z. Phys. C33 (1986) 1.

[11] H. J. Drescher, A. Dumitru, and M. Strikman Phys. Rev. Lett. 94 (2005) 231801, [hep-ph/0408073].

[12] K. Werner, F.-M. Liu, and T. Pierog Phys. Rev. C74 (2006) 044902, [hep-ph/ 0506232 ].

[13] S. Ostapchenko Phys. Rev. D83 (2011) 014018, [1010.1869].

[14] S. Ostapchenko, M. Bleicher, T. Pierog, and K. Werner Phys. Rev. D94 (2016) 114026 , [1608.07791].

[15] B. Andersson, G. Gustafson, and B. Soderberg Z. Phys. C20 (1983) 317.

[16] T. Sjöstrand Int. J. Mod. Phys. A3 (1988) 751.

[17] B. Andersson, G. Gustafson, and T. Sjostrand Phys. Scripta 32 (1985) 574.

[18] NA49 Collaboration, T. Anticic et al. Eur. Phys. J. C68 (2010) 1-73, [1 004 . 1889 ].

[19] A. Buckley, H. Hoeth, H. Lacker, H. Schulz, and J. E. von Seggern Eur. Phys. J. C65 (2010) 331-357, [0907.2973].

[20] A. Buckley et al. Comput. Phys. Commun. 184 (2013) 2803-2819, [1003. 0694 ].

[21] LHCf Collaboration, O. Adriani et al. Phys. Lett. B750 (2015) 360-366, [1 503.03505 ].

[22] A. Fedynitch et al. these proceedings, 2017.

[23] T. Bergmann et al. Astropart. Phys. 26 (2007) 420-432, [astro-ph / 0606564 ].

[24] T. Pierog, I. Karpenko, J. M. Katzy, E. Yatsenko, and K. Werner Phys. Rev. C92 (2015) 034906, [1306.0121]. 